\title{
Tableau digestif et syndrome confusionnel révélant une neurolistériose
}

\author{
Digestive and Confusional Symptoms Revealing a Neurolisteriosis
}

\author{
C. Lavoignet $\cdot$ M. Bousigues $\cdot$ H. Slimani \\ Reçu le 19 octobre 2018; accepté le 7 décembre 2018 \\ (C) SFMU et Lavoisier SAS 2018
}

\section{Introduction}

Les infections graves à Listeria monocytogenes sont rares et s'accompagnent volontiers d'un tableau digestif qui peut être au premier plan et masquer d'autres symptômes plus graves, neurologiques en particulier. Nous rapportons ici le cas clinique d'un patient qui s'est présenté aux urgences pour des troubles digestifs associés à une confusion fébrile, chez lequel a été mise en évidence une infection neuroméningée à $L$. monocytogenes.

\section{Observation}

Un patient de 70 ans a été admis aux urgences pour une diarrhée fébrile et des douleurs abdominales évoluant depuis quatre jours. Un traitement par métronidazole et ciprofloxacine avait été introduit en ville depuis trois jours devant une suspicion de colite infectieuse. Le tableau s'est compliqué d'un syndrome confusionnel avec une désorientation temporospatiale et une agitation psychomotrice d'installation progressive après 24 heures d'évolution. Le patient avait pour seul antécédent une cardiopathie ischémique. L'examen clinique retrouvait des paramètres vitaux normaux, avec une pression artérielle à $115 / 75 \mathrm{mmHg}$, une fréquence cardiaque à $78 / \mathrm{min}$ et une saturation en oxygène à $96 \%$. En revanche, le patient présentait une hyperthermie à $40,1{ }^{\circ} \mathrm{C}$. L'abdomen était sensible dans son ensemble, sans masse palpable et sans défense. Il n'existait pas de foyer pulmonaire. L'examen neurologique ne retrouvait en outre aucun signe déficitaire. Le patient ne se plaignait pas de céphalée, et il n'y avait pas de raideur de nuque, ni de photophonophobie. Le reste de l'examen clinique était sans particularité.

C. Lavoignet $\cdot$ M. Bousigues $\cdot$ H. Slimani $(\square)$

Service des urgences, hôpital Nord-Franche-Comté,

100, route de Moval, CS 10499 Trévenans,

F-90015 Belfort, France

e-mail : hakim.slimani@hnfc.fr
La biologie retrouvait un syndrome inflammatoire biologique avec une hyperleucocytose à $11,7 \mathrm{~g} / \mathrm{l}$ et une éosinopénie à $0 / \mathrm{mm}^{3}$. La protéine $\mathrm{C}$ réactive était à $151 \mathrm{mg} / \mathrm{l}$, la procalcitonine à $4,24 \mathrm{ng} / \mathrm{ml}$. Il existait une légère insuffisance rénale aiguë (créatininémie $123 \mu \mathrm{mol} / \mathrm{l}$, clairance de la créatinine à $56 \mathrm{ml} / \mathrm{min}$ par $1,73 \mathrm{~m}^{2}$ et urémie à $10 \mathrm{mmol} / \mathrm{l}$ sans trouble électrolytique). Le bilan hépatique était normal. Un scanner abdominal ne mettait pas en évidence de foyer infectieux profond, et le scanner cérébral ne retrouvait pas d'anomalie. Devant ce tableau de confusion fébrile sans point d'appel objectif, une ponction lombaire a été réalisée. Elle permettait d'extraire un liquide trouble, motivant la mise en route, immédiatement après la ponction, d'un traitement antibiotique probabiliste par céfotaxime à dose méningée, accompagné d'une injection intraveineuse de dexaméthasone $(10 \mathrm{mg})$ et d'aciclovir $(10 \mathrm{mg} / \mathrm{kg})$. L'analyse cytobiochimique du liquide céphalorachidien retrouvait 860 éléments nucléés par $\mathrm{ml}$, avec une formule panachée : $60 \%$ de polynucléaires et $40 \%$ de cellules mononucléées. Il existait également une hyperprotéinorachie à $3,73 \mathrm{~g} / 1$ et une hypoglycorachie à $3,4 \mathrm{mmol} / \mathrm{l}$ (pour une glycémie à $11,5 \mathrm{mmol} / \mathrm{l})$. L'examen direct était négatif. Il était alors décidé d'ajouter un traitement par amoxicilline à dose méningée pour couvrir Listeria, en raison de l'âge, et la présentation clinique atypique marquée par des troubles digestifs au premier plan. La culture du liquide céphalorachidien revenait positive après 24 heures, confirmant la suspicion diagnostique avec identification de L. monocytogenes. La gentamycine était alors ajoutée au traitement, et le céfotaxime était arrêté. Les hémocultures faites à l'admission restaient négatives. Le patient a été admis en service de réanimation. La déclaration à l'Agence régionale de santé (ARS) a été faite par le médecin réanimateur. La source alimentaire n'a pas été identifiée par l'ARS. L'évolution fut favorable avec une amélioration rapide sur le plan neurologique et une apyrexie obtenue en 36 heures. Le syndrome inflammatoire biologique était rapidement dégressif, et le patient est sorti d'hospitalisation après 16 jours d'hospitalisation sans séquelle. Il n'a pas été mis en évidence d'immunodépression en cours d'hospitalisation. 


\section{Discussion}

Le genre Listeria comporte six espèces dont l'espèce L. monocytogenes, la seule pathogène pour l'homme et les animaux [1]. Il s'agit d'un bacille intracellulaire à Gram positif, dont la principale voie d'infection est l'ingestion d'aliments crus infectés ( $99 \%$ des cas), comme les légumes crus, le lait cru, le poisson, la volaille et la viande. Contrairement à la plupart des agents pathogènes d'origine alimentaire, L. monocytogenes peut se développer aux températures du réfrigérateur [1]. La listériose est responsable en France chaque année d'environ 300 cas sporadiques (aucune épidémie n'a été identifiée en France depuis 2003 [1-3]. Le délai d'incubation après ingestion d'aliments contaminés est en moyenne de deux jours et jusqu'à neuf jours pour les formes neuroméningées [4]. La listériose peut se présenter sous deux formes : invasives et non invasives. Les formes non invasives sont rares et typiquement responsables d'un tableau de gastroentérite fébrile banale. Les formes invasives peuvent prendre trois présentations cliniques principales : les bactériémies (près de $50 \%$ des formes invasives), les atteintes du système nerveux central ou neurolistériose (près de $30 \%$ ), et les infections maternofœetales ou néonatales (près de $10 \%$ ) [2,3]. La méningoencéphalite est la forme clinique prédominante d'infection du système nerveux central. Les atteintes du parenchyme cérébral, habituellement associées à une méningite, sont classiquement localisées au tronc cérébral (rhombencéphalite avec atteinte des nerfs crâniens), mais cette présentation reste rare avec moins de $20 \%$ des neurolistérioses de la cohorte prospective française MONALISA [3]. L'encéphalite pure, les abcès cérébraux ou cérébelleux isolés sont exceptionnels et ne représentent que 1 à $5 \%$ des cas publiés dans la littérature [5]. Il existe des facteurs de risque devant faire évoquer une infection invasive à Listeria, tels que la grossesse particulièrement au troisième trimestre, les âges extrêmes de la vie (la période néonatale et l'âge supérieur à 60 ans), une immunodépression naturelle innée ou acquise, un traitement immunosuppresseur, les patients hémodialysés, les patients éthyliques chroniques ou avec une insuffisance hépatique chronique [1-3,6]. La neurolistériose est confirmée par la ponction lombaire qui retrouve habituellement un liquide cellulaire, polymorphe avec une hyperprotéinorachie. Une hypoglycorachie n'est retrouvée que dans 30 à $50 \%$ des cas, et l'examen direct du liquide céphalorachidien ne revient positif que dans environ un tiers des cas [3]. Les hémocultures sont positives dans environ $50 \%$ des cas. Le traitement de première intention comporte une association d'amoxicilline et de gentamicine. L. monocytogenes est naturellement résistante aux céphalosporines. En cas d'allergie aux bêtalactamines, il est recommandé d'utiliser l'association triméthoprime-sulfaméthoxazole en raison de sa très bonne diffu- sion dans le système nerveux central ainsi que dans le compartiment intracellulaire [3]. Des séquelles neurologiques à long terme sont observées pour $44 \%$ des patients dans les suites d'une neurolistériose [3] alors que la mortalité globale des infections neuroméningées à Listeria avoisine les 30 contre $45 \%$ dans les bactériémies [3]. Les facteurs associés à la mortalité dans la neurolistériose sont le cancer en cours, la défaillance multiviscérale, la comorbidité décompensée, la monocytopénie et la bactériémie associée à la neurolistériose [3]. L'utilisation précoce de dexaméthasone est recommandée en association avec l'antibiothérapie en cas de méningite purulente [7]. L'étude MONALISA a toutefois décrit une augmentation de la morbimortalité de la corticothérapie dans la neurolistériose [3]. Les résultats de cette étude confortent ainsi probablement la recommandation européenne d'arrêter la corticothérapie en cas de méningite purulente liée à un autre germe que Haemophilus influenzae ou Streptococcus pneumoniae [7].

\section{Conclusion}

L'infection neuroméningé à L. monocytogenes doit être évoquée devant l'association d'une confusion fébrile avec un tableau digestif, a fortiori chez les patients à risque. La ponction lombaire doit être réalisée rapidement afin de pouvoir introduire une antibiothérapie efficace sur Listeria.

Liens d'intérêts : les auteurs déclarent ne pas avoir de liens d'intérêts.

\section{Références}

1. Lomonaco S, Nucera D, Filipello V (2015) The evolution and epidemiology of Listeria monocytogenes in Europe and the United States. Infect Genet Evol 35:172-83

2. Goulet V, Hebert M, Hedberg C, et al (2012) Incidence of listeriosis and related mortality among groups at risk of acquiring listeriosis. Clin Infect Dis 54:652-60

3. Charlier C, Perrodeau E, Leclercq A, et al (2017) Clinical features and prognostic factors of listeriosis: the MONALISA (Multicentric Observational NAtional Study on LISteriosis and ListeriA) national prospective cohort study. Lancet Infect Dis 17:510-9

4. Goulet V, King LA, Vaillant V, DeValk H (2013) What is the incubation period for listeriosis? BMC Infect Dis 13:11

5. Mylonakis E, Hohmann EL, Calderwood SB (1998) Central nervous system infection with Listeria monocytogenes. 33 years' experience at a general hospital and review of 776 episodes from the literature. Medicine 77: 313-36

6. Mylonakis E, Paliou M, Hohmann EL, et al (2002) Listeriosis during pregnancy: a case series and review of 222 cases. Medicine 81: 60-9

7. Van de Beek D, Cabellos C, Dzupova O, et al (2016) ESCMID guideline: diagnosis and treatment of acute bacterial meningitis. Clin Microbiol Infect 22:37-62 